\title{
Packet Combining and APC using Decode and Forward Relay over Wireless Networks
}

\author{
Abhinandan Goswami \\ M.Tech student \\ Department of ECE \\ NIT Arunachal Pradesh
}

\author{
Srikanta Dash \\ M.Tech student \\ Department of ECE \\ NIT Arunachal Pradesh
}

\author{
Yang Saring \\ Assistant Professor \\ Department of ECE \\ NIT Arunachal Pradesh
}

\begin{abstract}
Decode and Forward (DF) is a type of opportunistic relay in which the packet is relayed only when the direct transmission of information from source to the destination fails. This results in bandwidth optimization of the overall system as well as considerable saving of resources by avoiding the unnecessary relay of information when it is not required. In this paper the authors have proposed a system using DF relay. By applying proper error correction techniques at the receiver side, it has been found that a corrected copy can be recovered from any erroneous receptions. Also the system offers much higher throughput and packet error rate (PER) performance, which is evident from the mathematical and simulation analysis made in the later part of this paper.
\end{abstract}

\section{Keywords}

Decode and Forward, Packet Combining, Error Correction, Aggressive Packet Combining, Majority Logic, Throughput, PER.

\section{INTRODUCTION}

With the advancements in the new generation of wireless technology, wireless networks are becoming very popular now a day. New generation wireless networks offer more flexibility, reliability and mobility with improved data rate and connectivity. Inherently, the wireless network is broadcast in nature and therefore it is possible for the wireless nodes or relays to forward each other's message in order to improve throughput, reduce the bit error rate (BER), improve capacity, coverage and reliability. This relay technique has been applied extensively in next generation wireless standards such as LTE, IEEE 802.16 (Wi-Max), Cognitive Radio and other such applications. In this work, we have undertaken an approach to propose an opportunistic relay scheme using Decode and Forward (DF) relay[1-3]. The single bit feedback from the destination indicating success or failure of transmission will decide the forward of message by a relay in the next phase. Here the transmission is usually performed in two phases or steps. In the first phase, the information from the source is being directly transmitted to the destination via a single path. If the receiver fails to receive the correct information, the relay transmits the message to the destination. If the transmission is successful in the first phase, then there is no need to transmit the message in the second phase. This leads to bandwidth optimization as well as efficient utilization of resources.

In this paper the authors have deployed Packet Combining(PC) [4] and Aggressive Packet Combining(APC) [5] techniques at the receiver side to rectify the errors and retrieve the correct packet. The transmitted packet from the source is overheard by all the relays which are in the range of the transmitter and stores the packet or information in its buffer. When a packet is successfully received by the receiver, it sends an ACK signal and in the case when it is erroneously received it sends a NACK signal. This ACK/NACK signal is also overheard by the relay stations. When an ACK signal is received by the relay station, it clears the packet stored in its buffer.[6-9]. If it receives a NACK signal, it retransmits the packet stored in its buffer instead of retransmission by the transmitter. If the relay nodes also does not transmit the correct packets in the subsequent phases, error correction techniques (PC/APC) are applied at the receiver side to rectify the errors and retrieve the correct packet. In the conventional cooperative diversity networks the relays transmit the source information to the destination irrespective of the channel condition. However, in the proposed method the relays transmit the information to the destination only when the direct transmission between source to destination fails. This failure in transmission can be recognized by the relays by hearing the negative acknowledgement or NACK signal. In this case the relay is used opportunistically based on the limited feedback from the receiver. [11-13]

\subsection{Contributions}

The aim to propose a DF relay model for wireless networks is to enhance the throughput efficiency of data transmission and also to utilize the available resources in an optimum manner leading to a highly reliable network in data transmission. With this in mind the following contributions have been made in this paper.

- By introducing this new scheme using DF relay a

highly efficient and reliable system for data transmission is proposed.

- Mathematical and simulation analysis is carried out to access the superiority of the proposed scheme over conventional error correction methods and results are depicted in the later part of this paper.

- This analysis clearly reflects that the proposed scheme indeed offers much better throughput efficiency and reduced PER than conventional error correction methods without relaying.

In the conventional error correction methods like PC and APC in which multiple copies of the same packet are transmitted from the source to the destination the throughput efficiency is quite low. Also in the conventional relaying methods used in broadcast communication all the transmitted copies are being sent at once, thus requiring more bandwidth which also results in wastage of available resources. The proposed scheme results in enhancement of throughput efficiency to a 
considerable extent as compared to conventional PC and APC scheme and also results in better utilization of available resources as the transmissions from the relay are made only on demand.

\subsection{Paper Organization}

The next section reviews the conventional PC and APC scheme and their error correction methods. Section 3 then illustrates the proposed scheme of deployment of DF relay in data transmission and Section 4 carries out a detail analysis of the scheme with illustrative examples. Section 5 depicts the mathematical analysis and Section 6 carries out experiments to obtain the throughput and the PER curves for the proposed scheme as compared to conventional methods of error correction and thereby establishing that the proposed scheme provides better throughput and error correction capability. Finally, section 7 concludes the paper and discusses future work.

\section{REVIEW OF PC AND APC SCHEME}

Chakraborty suggested a simple and elegant technique called Packet Combining scheme [4] in which the disjoint information contained in the erroneous copies of a packet is explored to obtain the correct copy.

It is assumed that an original packet " 11001000 " is to be transmitted from the source to the destination. The receiver receives the packet erroneously as " 11011000 " (The bits in error are marked in bold). The receiver requests for retransmission and buffers the erroneous copy. Say the second copy is also erroneously received by the receiver as "10001000". Chakraborty suggested that the receiver can locate the error positions by bit wise XORing the two received copies. The operation is illustrated below:

First Copy: 11011000

Second Copy: 10001000

After XORing: 01010000

The error locations are identified as $2^{\text {nd }}$ and $4^{\text {th }}$ bit from the left . Chakraborty suggested that the receiver can apply the brute force method to correct the error by changing the received " 1 " to " 0 " and vice versa followed by the application of error detection method in use. In the given example the average number of brute application will be $1 / 2$ and in general $2^{\mathrm{n}-1}$ if ' $\mathrm{n}$ ' bits are found in error.

Aggressive Packet Combining scheme is a modified form of MjPC (Majority packet combining). To illustrate this scheme let us consider that an original packet "11110001" is transmitted between the source and the destination. In APC three copies of each packet are sent from the sender to the receiver and bit by bit majority logic is applied on the received copies as follows:

First Copy: 11100001

Second Copy: 01110001

Third Copy: 10110001

Majority Logic: 11110001 which is the correct packet

In this way the receiver can obtain the correct packet through majority voting at the receiver side.

\section{PROPOSED SCHEME WITH DECODE AND FORWARD RELAY}

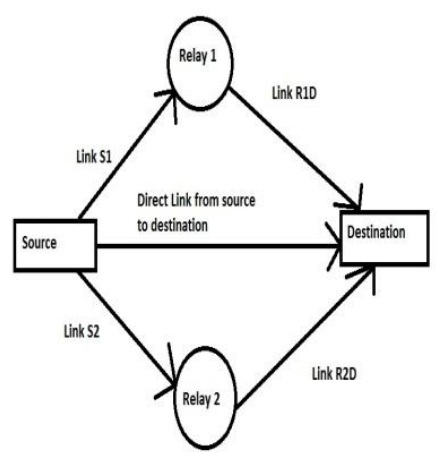

Fig 1. Proposed scheme with DF relay

The above figure (Fig. 1) illustrates a system with DF relay. The figure depicts a system with two DF relays, a source from where the packets will be transmitted and a destination where it will be received. The packet from the source is being transmitted to the destination via the direct link. The same packet is also overheard by Relay 1 and Relay 2 which are in the range of the transmitter. If the packet is successfully received by the receiver, it sends an ACK signal to the transmitter. This ACK signal will also be received by the two relays and they will clear the previous packet stored in their buffers. In the case where the packet is erroneously received by the receiver it will send a NACK signal back to the transmitter and stores the erroneous packet in its buffer. This NACK signal will also be overheard by the two relays and they will transmit their copies of the packet to the destination. The receiver will now have a total of three copies and it will apply APC technique using majority voting to recover the correct packet.

\subsection{CASE 1: When The Copies Of The Packets From The Relays Reaches The Destination Without Link Failure}

In receiving a negative acknowledgement (NACK) from the destination, the transmitter will wait for a specific time period before the next transmission. In the mean time the relays (Relay 1 and Relay 2) transmit their copies of the packet to the receiver through the links R1D and R2D. If these copies are also received with error, then the receiver will apply Aggressive Packet Combining (APC) technique by bitwise majority voting of the three erroneous copies to rectify the errors and to recover the correct packet. In the worst case if the correct packet is not recovered through APC it sends another NACK and the transmitter retransmits the packet.

\subsection{CASE 2: When A Copy Of Packet From A Relay Is Lost During Transmission Due To Link Failure.}

In the case when one copy of packet from either relay (Relay 1 or Relay 2) is lost during transmission, the receiver will have only two copies. First, the copy received through direct link and second, the copy through one relay. If the copy from the relay is also erroneous, then the receiver will apply the Packet Combining technique by bitwise XORing the two erroneous copies to identify the error locations. The receiver can apply the brute force method to correct the errors through bit by bit inversion of the received copies followed by error 
detection methods in use. If the receiver fails to recover the correct copy, then it sends another NACK to the transmitter and it retransmits the packet.

\subsection{CASE 3: When The Packet Is Lost During Transmission Through Direct Link.}

In the case when the packet transmitted through direct link does not reach the destination due to link failure, the transmitter will not receive any feedback or acknowledgement from the receiver. In Automatic Repeat Request (ARQ) scheme, the transmitter waits for a specific time period and if it does not receive any acknowledgement, the packet is retransmitted by the transmitter. But in this case instead of the transmitter, the copies of the packet stored in the relays (Relay 1 and Relay 2) are being transmitted to the destination. If both the copies are received with error, the receiver applies the Packet Combining technique to rectify the errors in order to recover the correct packet. In this way, by reducing the number of retransmissions from the transmitter, throughput of the overall system can be enhanced.

\section{ILLUSTRATIVE EXAMPLES OF THE PROPOSED SCHEME}

\subsection{Example for CASE 1}

Suppose the original packet to be transmitted is 10100011

At first, the packet is transmitted to the destination through the direct link. Let it be received as 10110011 which is in error. (The error location is marked in bold).

\begin{tabular}{|l|l|}
\hline Original Packet & $\begin{array}{l}\text { Packet received through } \\
\text { direct link }\end{array}$ \\
\hline 10100011 & 10110011 \\
\hline
\end{tabular}

The receiver will send NACK request back to the transmitter which will also be overheard by Relay 1 and Relay 2 . The transmitter will wait for a specified time period. In that period, both the relays will simultaneously transmit their copies to the destination which are received as

Relay 1 Copy: 10110011

Relay 2 Copy: 11100011

As both the received copies are also in error the receiver will apply aggressive packet combining (APC) by bitwise majority voting on the three received erroneous copies. The scenario is illustrated below:

\begin{tabular}{ll}
$1^{\text {st }}$ Copy: & 10110011 \\
$2^{\text {nd }}$ Copy: & 10110011 \\
$3^{\text {rd }}$ Copy: & 11100011 \\
\hline
\end{tabular}

\section{Majority Voting: 10110011}

The obtained packet through majority voting is also not the correct one. The receiver will search for the least reliable bits which are $2^{\text {nd }}$ and $4^{\text {th }}$ bit from left. Receiver then search for the correct bit patterns to generate the correct copy for the transmitted packet.

\subsection{Example for CASE 2}

Suppose the original packet to be transmitted is 1001

At first, the packet is transmitted to the destination through the direct link. Let it be received as 1011 which is in error
(The bits in error are marked in bold).

\begin{tabular}{|c|c|}
\hline Original Packet & Received through direct link \\
\hline 1001 & 1011 \\
\hline
\end{tabular}

The receiver will send NACK request back to the transmitter which will also be overheard by Relay 1 and Relay 2 . The transmitter will wait for a time period before retransmitting the packet. In the mean time the relays will transmit their copies to the destination. Say, the copy from relay 2 is lost during transmission and the receiver receives only the copy from relay 1 which is also received with error as 1000 .

\begin{tabular}{|c|c|}
\hline Packet send by Relay 1 & $\begin{array}{l}\text { Packet received at the } \\
\text { destination }\end{array}$ \\
\hline 1001 & 1000 \\
\hline
\end{tabular}

The receiver will apply packet combining technique by bitwise XORing the two erroneous copies to detect the error locations.

$1^{\text {st }}$ copy: 1011

$2^{\text {nd }}$ copy: 1000

XORing 0011

The error locations are identified as $3^{\text {rd }}$ and $4^{\text {th }}$ bit from left. The receiver will apply the brute force method by bit by bit inversion on the received copies to rectify the errors and obtain the correct copy.

\subsection{Example for CASE 3}

Suppose the original packet to be transmitted is 1110

At first, the packet is transmitted to the destination through the direct link. Due to link failure, the packet is not received at the destination. The transmitter and the relays wait for a specific time period and due to non receipt of the feedback or acknowledgement from the receiver, the relays transmits their copies to the destination instead of the transmitter.

\begin{tabular}{|l|l|}
\hline Original Packet & $\begin{array}{l}\text { Packets received through } \\
\text { relays }\end{array}$ \\
\hline 1110 & 1100 \\
& 1111 \\
\hline
\end{tabular}

As both the copies are received with errors, the receiver applies the Packet Combining technique by bitwise XORing the two erroneous copies to detect the error locations.

$1^{\text {st }}$ Copy: 1100

$2^{\text {nd }}$ Copy: 1111

XORing : 0011

The error locations are identified as $3^{\text {rd }}$ and $4^{\text {th }}$ bit from the left. The receiver applies the brute force method by bit by bit inversion on the received copies to rectify the errors in order obtain the correct copy.

\section{MATHEMATICAL ANALYSIS}

The probability of packet in error [10] is given by

$$
\text { PER }=\left(1-(1-\alpha)^{n}\right)^{k}
$$

Where ' $\alpha$ ' is the bit error rate (BER), ' $n$ ' is the number of bits in the packet, ' $\mathrm{k}$ ' is the number of copy being transmitted.

In PC scheme two copies of a packet are required, so $\mathrm{k}=2$ and therefore PER of PC scheme can be expressed as: [10] 


$$
P_{P C}=\left(1-(1-\alpha)^{n}\right)^{2}
$$

In APC scheme three copies of a packet are required, so $\mathrm{k}=3$ and therefore PER of PC scheme can be expressed as: [10]

$$
P_{A P C}=\left(1-(1-\alpha)^{n}\right)^{3}
$$

In CASE 1 of DFR scheme packets are transmitted through two different paths on demand. Therefore PER for this case can be defined as

$$
P_{D F R}=\left(1-(1-a)^{n}\right)^{3} * 1
$$

In CASE 2 of DFR scheme packets are also transmitted through three different paths on demand but one of the copies is lost during transmission. Therefore PER for this case can be defined as

$$
P_{D F R}=\left(1-(1-a)^{n}\right)^{2} * 1
$$

In CASE 3 of DFR scheme packets are transmitted through two relays only i.e. via two paths only when the direct transmission link fails or on the non receipt of acknowledgement from the destination. Therefore PER for this case can be defined as

$$
P_{D F R}=\left(1-(1-a)^{n}\right)^{1} *\left(1-(1-a)^{n}\right)^{1}
$$

The throughput of conventional PC scheme is: [10]

$$
\eta_{P C}=\frac{1-P_{P C}}{2+P_{P C}}
$$

The throughput of conventional APC scheme is: [10]

$$
\eta_{A P C}=\frac{1-P_{A P C}}{3+P_{A P C}}
$$

The throughput of the proposed scheme using DF relay for CASE

$$
\eta_{D F R=}\left(1 * \mathrm{P}_{\mathrm{DL}}\right)+\left(\frac{1-P_{A P C}}{1+P_{A P C}} * \mathrm{P}_{\mathrm{APC}}\right)
$$

The throughput of the proposed scheme using DF relay for CASE 2 is

$$
\eta_{D F R=}\left(1 * \mathrm{P}_{\mathrm{DL}}\right)+\left(\frac{1-P_{P C}}{1+P_{P C}} * \mathrm{P}_{\mathrm{PC}}\right)
$$

The throughput of the proposed scheme using DF relay for CASE 3 is

$$
\eta_{D F R}=\frac{1-P_{P C}}{1+P_{P C}}
$$

where $\mathrm{P}_{\mathrm{DL}}$ is the probability of receiving the successful packet through direct link, $\mathrm{P}_{\mathrm{PC}}$ is the probability of receiving the successful packet through $\mathrm{PC}$ scheme and $\mathrm{P}_{\mathrm{APC}}$ is the probability of receiving the successful packet through APC scheme.

\section{SIMULATION RESULTS}

The typical Bit Error Rate (BER) for wireless channel is as high as $10^{-2}$ to $10^{-4}$. The performances of the proposed DF Relay scheme and conventional PC and APC scheme is compared in terms of Packet Error Rate (lower PER indicates better correction capability) and throughput efficiency. We did experiment with packet size $n=128$ and simulated the result on MATLAB simulator. We studied the performance of the proposed scheme and conventional PC and APC over BER from $10^{-2}$ to $10^{-4}$ and portrayed the results in Fig. 2.1, Fig. 2.2, Fig. 3.1, Fig. 3.2 and Fig 3.3. We see that the proposed scheme has achieved significantly better performance than the conventional packet combining schemes. The throughput of the DF relays scheme approaches $100 \%$, which is evident from the fact that only one copy of each packet is sent from the transmitter which is broadcasted by the relays to the destination.

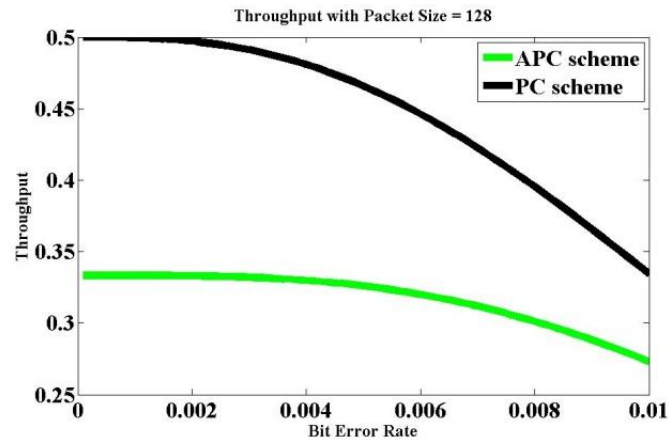

Fig 2.1 Throughput Comparison of PC and APC scheme

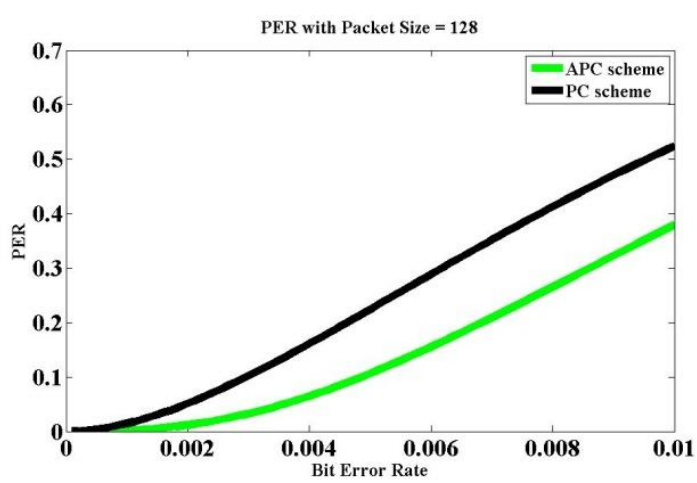

Fig 2.2 PER Comparison of PC and APC scheme

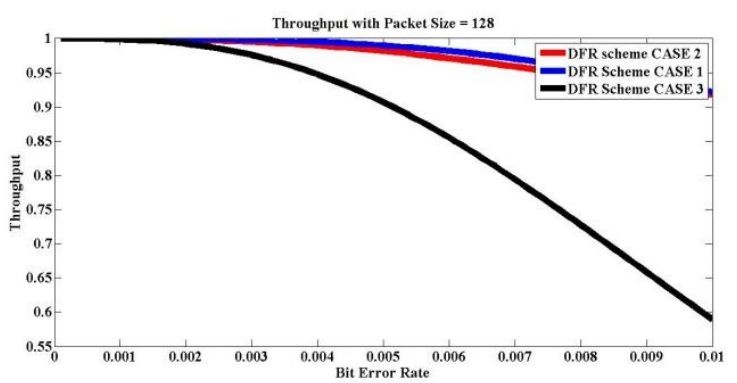

Fig 3.1 Throughput comparison of the proposed scheme

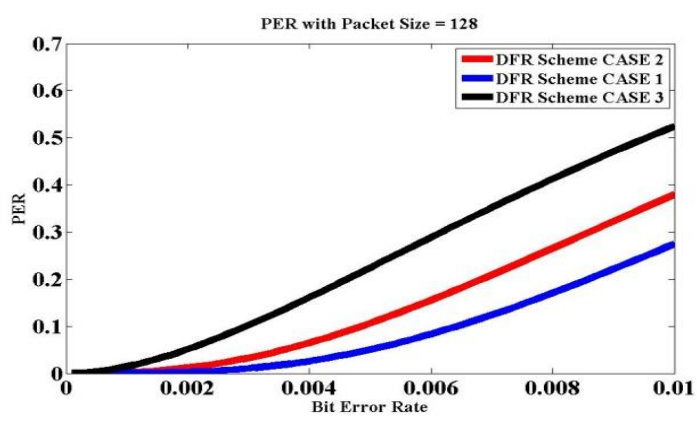

Fig 3.2 PER Comparison of the proposed scheme

From the figures 2.2 and 3.2 it can be seen that the packet error rate (PER) of the overall system reduces to a great extent 
as compared to the conventional PC and APC which makes the system fair enough for data transmissions over wireless networks.

\section{CONCLUSION AND FUTURE WORK}

In this paper the authors proposed a system to implement packet combining techniques using Decode and Forward (DF) relay for wireless networks. The proposed scheme is more bandwidth efficient than the conventional relay methodologies. As evident from the experimental results this technique offers much better throughput and reduced packet error rate. It can be seen that the proposed method improves the throughput by almost $50 \%$ as compared to conventional packet combining. It can be concluded that using DF relay scheme a highly efficient scheme for data transmission can be developed and proposed.

The future work related with this concept deals with increasing the transmission efficiency as well as improving the correction capability of the overall system. Also the time delay during transmission can also lead to a significant impact on the system performance, which will be thoroughly discussed in the future.

\section{REFERENCES}

[1] Rajendrakumar Patil, " On throughput performance of Decode and Forward Cooperative relaying with packet combining and ARQ", International Journal of Computer Networks and Communications (IJCNC), Vol 4. No. 3, May 2012.

[2] Yindi Jing, Hamid Jafarkhani, " Single and multiple relay selection schemes and their achievable diversity orders", IEEE transactions on wireless communications. (Volume-8, Issue-3), March 2009.

[3] Trung. Q. Duong, Vo Nguyen Quoc Bao, Hans-jurgen Zepernick, " On the performance of selection decodeand-forward relay networks over Nakagami-m fading channels", IEEE Communications Letters, (Volume-13, Issue-3), March 2009.

[4] Shyam S. Chakraborty et al, "An ARQ Scheme with Packet Combining," IEEE Comm Letters, vol. 2, No. 7, pp. 200-202, July'95.
[5] Yiu-Wing LEUNG, "Aggressive Packet Combining for Error Control in Wireless Networks," trans. Comm Vol. E83, No. 2, pp38-385, Feb'2000.

[6] Shyam S Chakraborty et al, "An Adaptive ARQ Scheme with Packet Combining for Time Varying Channels," IEEE Comm Letters, Vol 3, No. 2, pp 52-54, Feb 1999

[7] C T Bhunia, "Modified Aggressive Packet Combining Scheme," Pre-print, ICTP, Italy, IC/2010/037, pp. 1-10, June 2010.

[8] C.T. Bhunia, "Several Modifications of Aggressive Packet Combining Scheme for Wireless Network", Proc. of the IEEE International Conference on Computer, Information and Telecommunication Systems (CITS), 2012.

[9] C T Bhunia, "A Few Modified ARQ Techniques", Proceedings of the International Conference on Communications, Computers \& Devices, ICCCD-2000, 14-16 Decedmber'2000, IIT, Kharagpur, India, Vol.II, pp. 705-708.

[10] C T Bhunia, IT, Network \& Internet, New Age International Publishers, India, 2005.

[11] Ranita Khumukcham, Abhinandan Goswami, Yang Saring, "Four new protocols for achieving better correction capability of APC scheme", International Conference of Communication and Signal Processing (ICCSP-2015), Melmaruvathur, Tamil Nadu, India.

[12] Ranita Khumukcham, Abhinandan Goswami, Yang Saring, "Combined APC-PC scheme for random and time varying channels" , International Conference of Communication and Signal Processing (ICCSP-2015), Melmaruvathur, Tamil Nadu, India.

[13] Abhinandan Goswami, Yang Saring, C.T.Bhunia ,: "Aggressive Packet Combining Scheme In Multipath Routing To Achieve Higher Throughput And Error Correction Rates", International Journal of Electrical, Electronics and Data Communication (IJEEDC), Volume-4,Issue-1,pp 26-30,2016. 